\title{
transformación de dragas ancladas, en otras de suckión en marcha
}

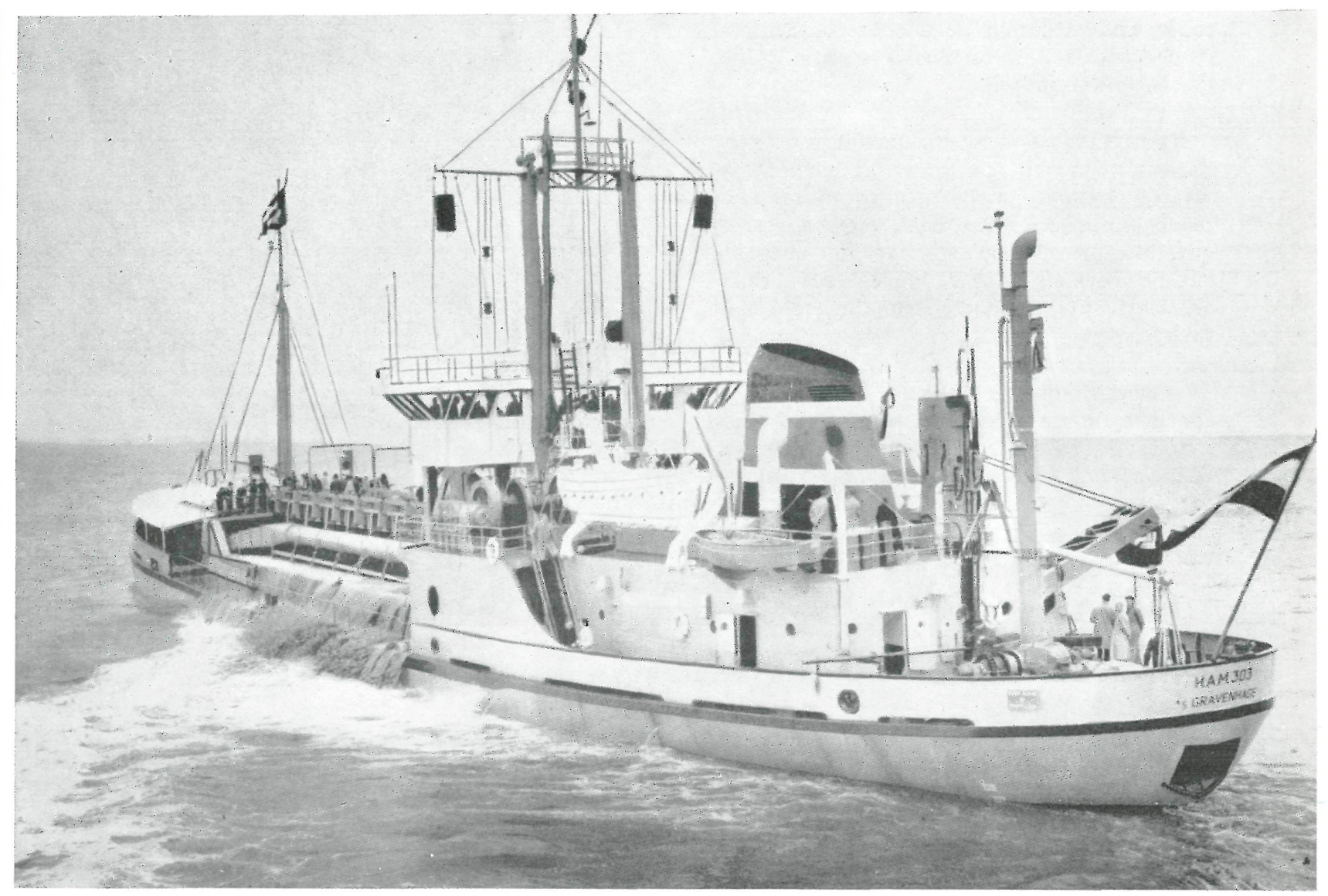

simopsis

$555-11$

El creciente desarrollo del tráfico marítimo impuso mayores calados en las vías de navegación. Para cumplir estos requisitos ha sido imprescindible un perfeccionamiento en los trabajos realizados por las dragas. Los avances obtenidos en la construcción de las dragas de succión en marcha han permitido un empleo más intenso de éstas, al poderse efectuar con ellas en la actualidad cuanto al número, situación y accionamiento de los tubos de subles. Las características de las dragas de succión en marcha, en que todos pueden juzgarse tan satisfactorios que hacen, en muchos casos, convenientes las reconstrucciones y transformaciones de dragas del antiguo sistema en dragas de succión en marcha.

El creciente desarrollo del tráfico marítimo y el aumento de calados, exige mayores y mejores condiciones de navegabilidad en las vías marítimas.

La maquinaria empleada para la conservación y mejora de estas características está sometida actualmente a unas exigencias crecientes. De acuerdo con estas necesidades están siendo empleadas nuevas técnicas dirigidas, bien a la creación de nuevos tipos, bien a la transformación de las máquinas existentes en modelos de distinta concepción de trabajo.

Caso concreto interesante es el de una draga construida en el año 1939, y que se proyectó para trabajar como draga de succión anclada en la conservación de la ría de Rotterdam. Este modelo, dispuesto para efectuar su trabajo anclado, fue superado en su capacidad dragadora por las crecientes necesidades, ya que se necesitaban efectuar trabajos en mar abierto, en los que frecuentemente el mal tiempo impedía su realización, así como un aumento de la profundidad de trabajo y de la maniobrabilidad de la draga para no interferir el tráfico marítimo. 
Todos estos inconvenientes aconsejaron el empleo de una draga de succión en marcha; pero la decisión adoptada consistió, no en la adquisición ni proyecto de una nueva draga, sino en la transformación de la existente, aprovechando las buenas condiciones de conservación de la misma, en una draga de succión en marcha.

Completada la transformación, esta draga, equipada con dos tubos de succión laterales flexibles, puede aspirar en marcha hasta una profundidad de 18 metros, y asegurando un tiempo efectivo de dragado cuatro veces superior al que le era normai anteriormente, al poder trabajar con mal tiempo.

Naturalmente, la solución en este caso concreto difiere de otras experiencias, pero sus resultados, y el desarrollo que en estos últimos años han experimentado los diver-

sos tipos de dragas de succión, hacen interesante una recopilación de las principales características de este tipo de dragas y de las mejoras esenciales características de la succión en marcha.

\section{Estudio compurativo de este tipo de dragus}

Característica común de estos modelos es el arrastre por el fondo de uno o más tubos de succión dotados del cabezal apropiado para verificar la absorción del material y la cántara para la recogida de los productos dragados.

Las mejores condiciones de navegabilidad de que se pretende dotar a este tipo de dragas, así como la maniobrabilidad exigida han hecho que la mayoría de ellas estén dotadas de dos hélices y dos timones (fig. 1).

Las limitaciones que se sufrían por ser únicamente capaces de absorber fangos han sido ampliamente superadas, pudiéndose realizar en la actualidad dragados de arcillas y arenas mediante absorción.

Las velocidades normales de transporte son de 9 a 13 nudos, oscilando las de trabajo entre dos y tres millas por hora.

Las ventajas características de las dragas de succión, sobre las de trabajo anclado, son las siguientes:

Ausencia de cables de anclaje, que limiten la vía navegable; la automoción de las mismas, que permite el fácil traslado de una a otra zona de dragado en cualquier momento; la posibilidad de trabajar en mar abierto y bajo estados de mar variables, y la formación en el fondo de surcos uniformes en contraposición con los pozos formados por el dragado de anclaje.

\section{Candetenisticas distrintivas}

Las experiencias obtenidas en el empleo y construcción de las dragas de succión en marcha han conducido a la ejecución de tipos de diversas características, con objeto de que las mismas puedan amoldarse, con facilidad, bien a un trabajo de condiciones prefijadas y características, o bien permitir una amplia gama de aplicaciones en circunstancias variadas. 


\section{Núnemero, situcución y accioncumiento de los tubos}

El número y situación de los tubos de succión de que está dotado un determinado tipo de draga, influye no solamente en el rendimiento de la misma y en la cantidad de productos dragados, sino en las características de trabajo, navegabilidad y maniobra del modelo.

Normalmente, son uno o dos los tubos de succión de que está dotada una draga de succión en marcha. Las que llevan dos tubos, por estar sometidas a esfuerzos simétricos, presentan, además de un mayor volumen de productos dragados, una estabilidad mejor en el rumbo. En cambio, las que poseen un tubo único ofrecen una instalación más simplificada y una cámara de máquinas menor que permite mayor espacio disponible; además, la estabilidad y características de trabajo también son buenas en este tipo, por lo que en la elección de una draga no podrá considerar como factor exclusivo el número de tubos que presenten.

Más importancia tiene la situación, montaje y accionamiento de los tubos de succión.

Normalmente, las dragas de succión en marcha, como consecuencia de las elevadas características exigidas de maniobrabilidad, disponen de dos timones y dos hélices, por lo que comúnmente, como mínimo, serán dos los motores de propulsión empleados.

En dragas de un solo tubo de succión existe algún tipo que emplea los dos motores para la navegación, accionando el motor de babor (fig. 2) el tubo de succión durante el dragado y permaneciendo el de estribor en la propulsión del barco.

Una construcción más apropiada es la de aquellos tipos dotados de tres máquinas (fig. 3): dos para asegurar la propulsión y una tercera para el accionamiento de la bomba de dragado; la potencia propulsora durante las operaciones de dragado es mayor, así como también es mejor la maniobrabilidad que en las dragas del tipo descrito anteriormente, permitiendo además esta instalación disponer de una reserva de potencia en caso necesario.

Dentro de los tipos de draga que disponen de dos tubos de succión laterales, la situación de los mismos puede ser delante o detrás de la cántara, siendo la colocación un significado distintivo por las diferentes características que ofrecen.
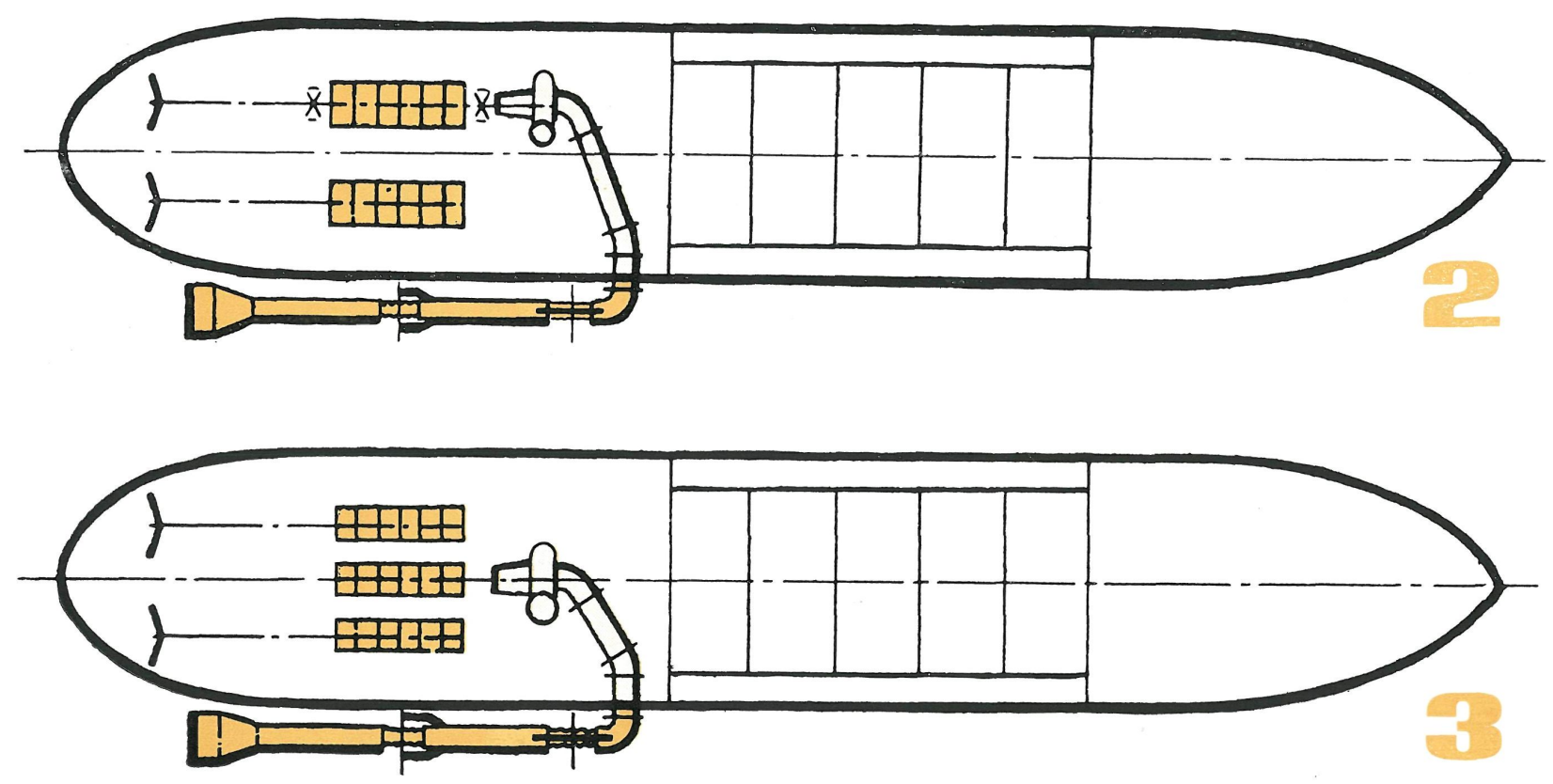

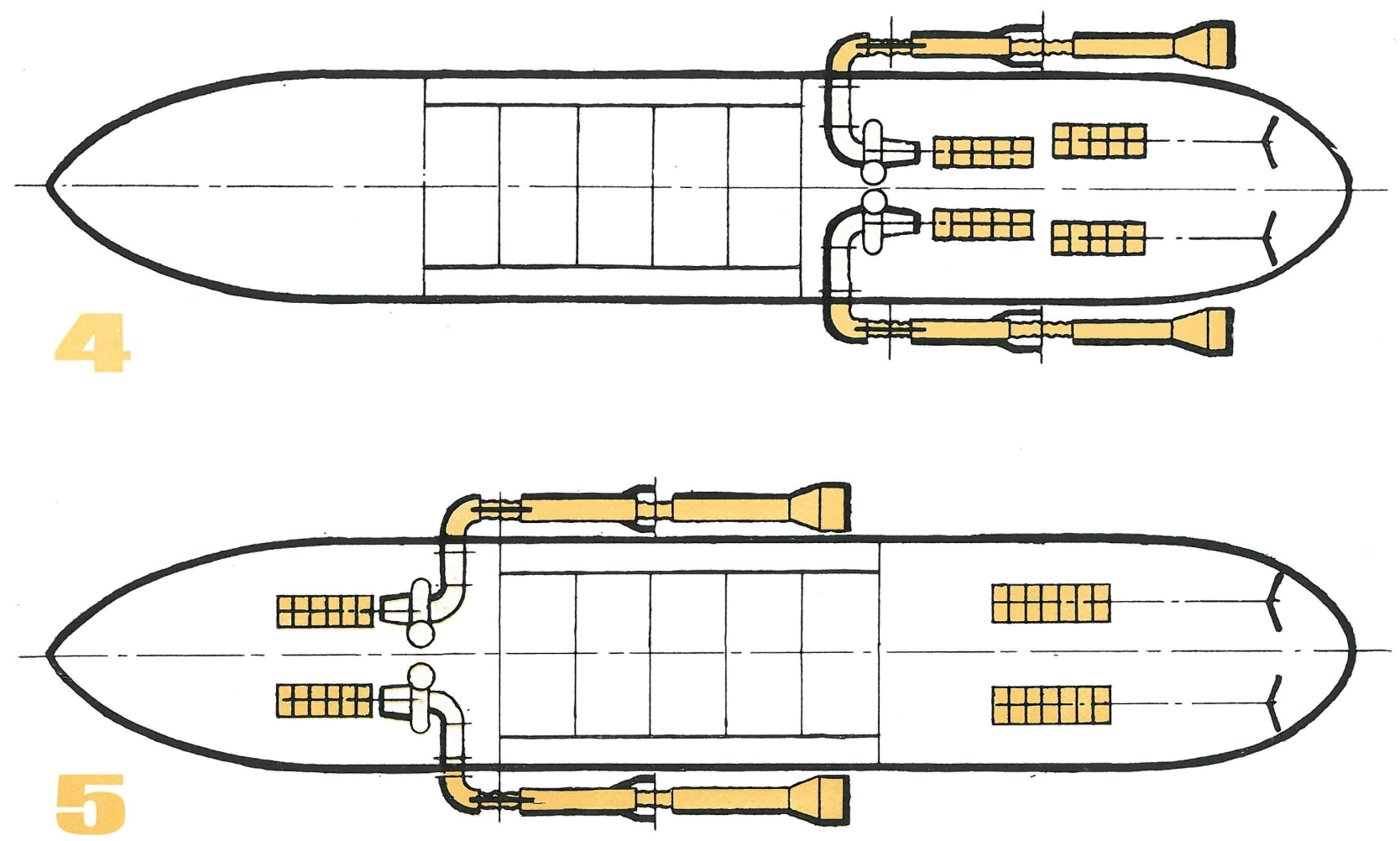

Las dragas que tienen los tubos de succión detrás de la cántara (fig. 4), presentan la ventaja de tener una sala de máquinas única, lo que permite mayor facilidad de mando y vigilancia. En contraposición, la eslora necesaria es mayor y la distribución del peso a bordo más desequilibrada que en los modelos en los que los tubos de succión están situados a proa de la cántara.

En éstos son necesarias dos salas de máquinas (fig. 5), si bien el mayor inconveniente subsiguiente a esta duplicidad, queda subsanado prácticamente mediante instalaciones diesel-eléctricas que compensan el mayor consumo de combustible por caballo, al permitir, en cambio, que las bombas estén bajo el mando del puente o la sala de motores, con lo que el patrón tiene a su alcance todos los elementos de mando de la draga, no necesitándose más que una guardia en la cámara de proa.

La colocación a proa de los tubos de succión permite una mejor uniformidad en el reparto de pesos y la fácil colocación de los tubos a lo largo de la cántara, dejando despejada la cubierta de popa.

\section{Resumem}

Los estudios efectuados y las mejoras técnicas introducidas, han permitido conseguir efectuar recientemente trabajos y conseguir rendimientos mediante el empleo de dragas de succión que se juzgaban hace poco imposibles de lograr.

Por otra parte, los resultados logrados permiten, con resultados satisfactorios, reconstruir o transformar dragas de los antiguos tipos, con el consiguiente ahorro y mejora de los rendimientos y trabajos efectuados.

\section{CHINCHILLA}

(Agradeciendo sinceramente la información y fotografías facilitadas por la I. H. C. Holland.) 


\section{Trecinsformention de dreagues}

Le développement croissant du trafic maritime a imposé une plus grande profondeur des voies de navigation. Afin de pouvoir faire face à cette nécessité, un perfectionnement des travaux exécutés par les dragues a été indispensable.

Les progrès réalisés dans la construction des dragues à succion en marche ont permis leur utilisation plus intense, car l'on peut actuellement effectuer avec elles des travaux jusqu'à présent impossibles à exécuter.

Les caractéristiques des dragues à succion en marche, tant en ce qui concerne le nombre, la situation et le maniement des tubes de succion, n'influent pas décisivement sur le rendement de chaque type, puisque tous ces types peuvent être considérés si satisfaisants qu'ils exigent, en beaucoup de cas, les reconstructions et les transformations de drague de l'ancien système en dragues à succion en marche.

\section{Trumssformantion of Dredging Equipument}

With the large increase in shipping traffic and size of ships it has become necessary to deepen the navigation lanes in harbours and estuaries.

To meet this the dredging operations have also had to be greatly improved and modified.

The advancement attained in the construction of non stationary suction dredging equipment has made it possible to use this technique on a much wider scale, and so undertake programs which up to recently seemed almost impossible.

The particular design of non stationary suction dredges, as regards the number of suction tubes and their mode of operation, does not have a decisive influence on the overall performance of such equipment, since all such designs can be regarded as highly efficient. Hence it is very often regarded as advisable to reconstruct old type dredging equipment and adapt it to the new technique.

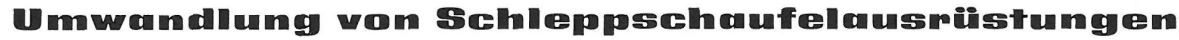

Die steigende Entwicklung des Schiffsverkehres verlangte einen grösseren Tiefgang bei den Schiffahrtswegen. Um diese Erfordernisse zu erfüllen, war eine Vervollkommnung bei den von den Schleppschaufelausrüstungen ausgeführten Arbeiten unerlässlich.

Die erzielten Fortschritte bei dem Bau der beweglichen Saugbagger haben eine intensivere Anwendung derselben erlaubt, da man mit ihnen gegenwärtig Arbeiten durchführen kann, die man bis vor kurzem als unmöglich ansah.

Die Eigenschaften der beweglichen Saugbagger bezüglich der Anzahl, Lage und des Antriebes der Saugrohre üben keinen entscheidenden Einfluss auf die Leistung jeder Art aus, da man alle als so zufriedenstellend beurteilen kann, dass sie in vielen Fällen den Wiederaufbau und die Umwandlungen der Bagger des alten Systems in bewegliche Saugbagger als angebracht erscheinen lassen. 